Max Ramírez*, Jose Rogan, Juan Alejandro Valdivia, Alejandro Varas, and Miguel Kiwi

\title{
Diversity Characterization of Binary Clusters by Means of a Generalized Distance
}

DOI 10.1515/zpch-2015-0716

Received October 15, 2015; accepted January 25, 2016

\begin{abstract}
We characterize, by means of the definition of a generalized distance, the differences and similarities between binary nanoclusters. To define analytically, and to compute numerically this distance, we have generalized an original concept that was introduced for pure clusters. Since the diversity of cluster conformations grows exponentially with their size, and becomes even larger when the cluster atoms are of more than one species, we limit our attention to small ones. Thus, to illustrate and analyze our distance definition we characterize the Lennard-Jones (LJ) minimum energy conformations of two- and threedimensional (2D and 3D) binary clusters, for $5 \leq N \leq 12$, where $N$ is the number of atoms of the cluster. In addition, when varying the LJ potential parameters, we find that the number of minima decreases as the range of the potential of one of the species is increased, and confirm that minimal energy conformations adopt a well defined core-shell configuration.
\end{abstract}

Keywords: Binary Nanocluster Structures, Nanocluster Diversity.

PACS: $61.46 .-\mathrm{w}, 61.46 . \mathrm{Bc}$

Dedicated to Professor Michael Springborg on the occasion of his $60^{\text {th }}$ birthday

\footnotetext{
*Corresponding author: Max Ramírez, Departamento de Física, Facultad de Ciencias, Universidad de Chile, Santiago, Chile, and Centro para el Desarrollo de la Nanociencia y Nanotecnología, CEDENNA, Santiago, Chile, e-mail: mramirez@fisica.ciencias.uchile.cl Jose Rogan, Juan Alejandro Valdivia, Miguel Kiwi: Departamento de Física, Facultad de Ciencias, Universidad de Chile, Santiago, Chile, and Centro para el Desarrollo de la Nanociencia y Nanotecnología, CEDENNA, Santiago, Chile

Alejandro Varas: Nano-Bio Spectroscopy group, Universidad del País Vasco UPV/EHU, CFM CSIC-UPV/EHU-MPC and DIPC, Avenida de Tolosa 72, E-20018 Donostia/San Sebastián, Spain; and ETSF Scientific Development Centre, Avenida de Tolosa 72, E-20018 Donostia/San Sebastián, Spain
} 


\section{Introduction}

For more than a decade bimetallic clusters have attracted much attention, because of their potential applications in catalysis, optical, magnetic, electric and mechanical nanodevices. The possibility of fine-tuning their properties is enhanced when both size and composition are varied. However, the structural characterization, necessary for a precise understanding of their properties, becomes a problem, since the enormous amount of structural conformations of a pure cluster is significantly increased by the combinatorial possibilities of binary ones [1-3]. Therefore, the search for minimal energy configurations has to be approached following a strategy that i) insures the diversity of the local minimal energy conformations, that is, how many non-equivalent configurations are obtained as the two species are mixed; and, ii) balances reasonable computation times and the "certainty" of not loosing potentially important conformations.

Binary Lennard-Jones (BLJ) clusters have been studied for some time. Doye and Meyer [4] already in 2005, using Basin-Hopping [5], found global minimum configurations for BLJ clusters of up to 100 atoms. Later on Molayem et al. investigated the stability of $\mathrm{Ni}_{m} \mathrm{Ag}_{n}$ bimetallic nanoalloys for $2 \leq m+n \leq 60$ also using basin hopping [6]. Cassioli et al. [7] found new minimal energy conformations and confirmed results found much earlier by Munro et al. [8]. Dieterich and Hartke [9] analyzed 38 atom binary clusters, finding stable minima for fcc like conformations. Kolossváry and Bowers [10] found 17 new putative minima for these type clusters. Marques and Pereira [11] implemented a hybrid embedded atom approach with which they found a new global minimum of an $N=38$ binary, for $R=\sigma_{B B} / \sigma_{A A}=1.05$, where $\sigma_{A A}$ and $\sigma_{B B}$ are related to the position of the minima of the respective LJ potentials. Sicher et al. [12] used a modified minimal hopping scheme to find a large amount of new minima. Tao et al. [13] implemented a modified heuristic algorithm to discover 12 new putative minima. Rondina and Da Silva [14] modified the Basin-Hopping Monte Carlo to study BLJ clusters for $5 \leq N \leq 100$, and obtained new results for $31 \leq N \leq 94$. Very recently Takeuchi [15] explored minimal energy configurations for the rather large values of $1.4 \leq R \leq 2.0$ finding complicated structural growth patterns.

In this paper we study the configuration diversity of the local minimal energy conformations. In other words how to accurately discriminate between two nonequivalent structures. This problem has been studied mainly for single species clusters and for periodic systems, which has led to the development of several strategies [16-20]. We now examine some of them, and choose the one best fitted for generalization to include several atomic species. 
In this context the characterization of two conformations as equal or different is a key element. Lee et al. [16] introduced the following definition for the distance between two configurations, $\alpha$ and $\beta$, of a pure cluster:

$$
d(\alpha, \beta)=\sum_{m} m\left[2\left|H_{\alpha}(1, m)-H_{\beta}(1, m)\right|+\left|H_{\alpha}(2, m)-H_{\beta}(2, m)\right|\right],
$$

where $H_{\alpha}(1, m)\left[H_{\alpha}(2, m)\right]$ is the histogram of the number of cluster atoms that have $m$ atoms in the first [second] shell of the configuration $\alpha$. To specify the shell radii they used the nearest-neighbor and next-nearest-neighbor bulk distances. However, in this way the first and second neighbor distances are rather arbitrarily defined.

Cheng and Fournier [17] instead put forward a distance based on physical descriptors. It incorporates six of them, that correspond to structural parameters and they are the following: the coordination of an atom $c_{k}$ as the number of atoms inside a sphere of radius $d_{\text {max }}$, centered around atom $k$. Four descriptors $D$ are based on $c_{k}$; the average $\left(D_{1}\right)$, the mean square deviation $\left(D_{2}\right)$, the minimum $\left(D_{3}\right)$, and the maximum $\left(D_{4}\right)$, of $c_{k}$. The remaining two $\left(D_{5}\right.$ and $\left.D_{6}\right)$ measure the deviation from spherical symmetry, towards oblate or prolate structures, and are given by

$$
\begin{aligned}
& D_{5}=\frac{\left(I_{c}-I_{b}\right)^{2}+\left(I_{b}-I_{a}\right)^{2}+\left(I_{a}-I_{c}\right)^{2}}{I_{a}^{2}+I_{b}^{2}+I_{c}^{2}}, \\
& D_{6}=\frac{2 I_{b}-I_{a}-I_{c}}{I_{a}},
\end{aligned}
$$

where $I_{x x}, I_{y y}$ and $I_{z z}$ are the eigenvalues of the inertia tensor ordered from largest to smallest, $\left(I_{a}, I_{b}\right.$ and $\left.I_{c}\right)$, and thus satisfy $I_{a} \geq I_{b} \geq I_{c}$.

Next, the descriptor distance $d(\alpha, \beta)$ between the configurations $\alpha$ and $\beta$ is defined as

$$
d(\alpha, \beta)=\left[\sum_{\ell=1}^{6} \frac{\left(D_{\ell}^{\alpha}-D_{\ell}^{\beta}\right)^{2}}{\left(D_{\ell, \max }-D_{\ell, \min }\right)^{2}}\right]^{1 / 2},
$$

where $D_{\ell, \text { max }}\left(D_{\ell, \min }\right)$ is the maximum (minimum) of the descriptor $\ell$ among the $\alpha$ and $\beta$ clusters. The denominator of Equation (4) normalizes the distance, to insure that all descriptors have the same weight. Rogan et al. [18] based on moments of inertia of the clusters, showed that this distance is useful, for example to discriminate among prolate and oblate conformations. In 2013 Goedecker et al. [19] introduced an interesting and novel distance concept based on differential geometry concepts. 
The distance put forward by Grigoryan et al. [20] in 2003 has been used successfully in minimization algorithms, like the one described by Rogan et al. [21, 22]. Analytically, this distance reads

$$
D(\alpha, \beta)=\left[\frac{2}{N(N-1)} \sum_{i=1}^{N(N-1) / 2}\left(d_{i}^{(\alpha)}-d_{i}^{(\beta)}\right)^{2}\right]^{1 / 2},
$$

where, $d_{i}^{(\alpha)}$ is the sorted list of $N(N-1) / 2$ interatomic pair distances of cluster $\alpha$ (and similarly for $\beta$ ). $D(\alpha, \beta)$ has the significant advantage of being translationally and rotationally invariant, and furthermore it is fast and simple to evaluate.

Since the main goal of this paper is to study and characterize the diversity of the minimal energy of binary nanocluster conformations, we limit our examples to rather small clusters. In particular, we employ the ones obtained from the implementation of a BLJ potential. Our contribution is organized as follows: after this introduction, in Section 2 we provide details of the computational methods that we use. In Section 3 we generalize the distance between clusters concept of Equation (5) to properly describe binary systems, and define the BLJ potential. In Section 4 we present our results and the paper is closed with Section 5, where we summarize and draw the main conclusions.

\section{Computational methods}

Rogan et al. [21] proposed a strategy to find a diverse set of low lying local minima, as well as the global minimum. To do so, the fast inertial relaxation engine (FIRE) algorithm was massively used as an efficient local minimizer [23]. This procedure turns out to be quite good to reach the global minimum, and also most of the local minima. We have chosen to use the FIRE algorithm to obtain the minimal energy structures, because it is strictly local, it does not get stuck in transition states, and it is very fast. Our procedure, to obtain the energy minima for an $N$ atom cluster, and which is somewhat different from other FIRE applications, is setup by generating at random a set of $3 \mathrm{~N}$ coordinates, in a cubic box of edge length $3.75 \AA$ A, which we denominate the "initial configuration" or "seed". Each one of these seeds is allowed to evolve by means of the FIRE algorithm, until the largest absolute value of the force, acting on every single atom, is less than a predetermined required accuracy, in this case $10^{-10} \epsilon / \sigma$. We repeat this process $10^{7}$ times for each cluster. Then, by means of an extension of the similarity criterion put forward by Grigoryan and Springborg [20], we obtain the final set of different minima for a given $N$, a given concentration and a given BLJ potential, which is controlled by changing a single parameter. 


\section{Binary clusters}

\subsection{The distance between binary clusters}

While our main objective is to study the diversity of binary systems, we first have to develop the concept of distance. Therefore, we now extend the distance put forward by Grigoryan et al. [20] to describe a cluster with more than one atomic species. To do so we order the cluster interatomic distances in a similar way as Valle and Oganov [24, 25]. That is, we first list, from largest to smallest, the $N_{A A}$ separations of the atoms of species $A$ of the conformation $\alpha$, denoted as $d_{i}^{(\alpha, A)}$; next we do the same for species $B$. Finally, we also order the $N_{A B}$ interatomic distances between the two different species. This creates a new configuration distance vector of $N_{A A}+N_{B B}+N_{A B}=N(N-1) / 2$ components. Analytically, the distance between conformations $\alpha$ and $\beta$ is given by

$$
\begin{aligned}
D(\alpha ; \beta)= & \sqrt{\frac{2}{N(N-1)}}\left[\sum_{i=1}^{N_{A A}}\left(d_{i}^{(\alpha, A)}-d_{i}^{(\beta, A)}\right)^{2}+\sum_{i=1}^{N_{B B}}\left(d_{i}^{(\alpha, B)}-d_{i}^{(\beta, B)}\right)^{2}\right. \\
& \left.+\sum_{i=1}^{N_{A B}}\left(d_{i}^{(\alpha, \overline{A B})}-d_{i}^{(\beta, \overline{A B})}\right)^{2}\right]^{1 / 2},
\end{aligned}
$$

where $\overline{A B}$ denotes the geometrical distance between different species atoms, and the criterion for two binary clusters being equal is that $D(\alpha ; \beta) \leq D_{c}$, where $D_{c}$ is a critical value. Furthermore, this generalization of the distance by Grigoryan et al. [20] can be readily extended to clusters of three or more species, and for any number of atoms.

\subsection{Binary Lennard-Jones potential}

To test our procedure we limit our attention to BLJ clusters. The species are labeled as $A$ or $B$, and $N=N_{A}+N_{B}$. Atoms $i$ and $j$ interact through the pair potential

$$
v(i, j)=4 \varepsilon_{S, S^{\prime}}\left[\left(\frac{\sigma_{S, S^{\prime}}}{r_{i j}}\right)^{12}-\left(\frac{\sigma_{S, S^{\prime}}}{r_{i j}}\right)^{6}\right],
$$

where $S$ and $S^{\prime}$ label the atomic species ( $S$ and $S^{\prime}$ correspond to species $A$ or $B$ ), $\varepsilon_{S, S^{\prime}}$ is the depth of the potential well, and $2^{1 / 6} \sigma_{S, S^{\prime}}$ is the equilibrium distance. Here and throughout we report our energies in units of $\varepsilon$, and lengths in $\sigma$ units. 
The total energy of the cluster is given by

$$
E=\sum_{i<j} v(i, j) .
$$

Moreover, as already implemented by several authors $[4,7,11]$, we adopt $\varepsilon_{A A}=$ $\varepsilon_{B B}=\varepsilon_{A B}=\varepsilon_{B A}=1$, and

$$
\sigma_{S, S^{\prime}}=\left\{\begin{array}{cl}
\sigma_{A A} & \text { if } S=S^{\prime}=A, \\
\sigma_{B B} & \text { if } S=S^{\prime}=B, \\
\frac{\sigma_{A A}+\sigma_{B B}}{2} & \text { if } S \neq S^{\prime} .
\end{array}\right.
$$

Additionally, if we adopt $\sigma_{A A}=1$, and define $R=\sigma_{B B} / \sigma_{A A}$, we have

$$
\sigma_{S, S^{\prime}}=\left\{\begin{array}{cl}
1 & \text { if } S=S^{\prime}=A, \\
R & \text { if } S=S^{\prime}=B, \\
\frac{1+R}{2} & \text { if } S \neq S^{\prime} .
\end{array}\right.
$$

If we assume $T=\epsilon_{A A} / \epsilon_{B B} \neq 1$ we can investigate the effects due to different potential depths. In fact, we report below on calculations for $R=1.0, T=1.0 \mathrm{in}$ the majority of cases. However, we also explored the case $R=1.0, T=1.5$ for a specific example.

\section{Results}

As already discussed the problem of the diversity of conformations of binary clusters seems far from solved, thus allowing new ideas and techniques to be developed and tested. As already proposed in Rogan et al. [21], we present both 3D and 2D minimum energy configurations, the latter being specially useful when discussing small $N$ clusters which may assume planar conformations, as it is well known that $\mathrm{Au}$ for example, for $N \leq 12$ is planar, a result that DFT treatments yield but that phenomenological potentials do not obtain [26, 27]. Here we calculate the diversity of the sets of minima as a function of $R$. We investigate clusters of $5 \leq N \leq 12$ atoms for $R=1.0,1.4,1.6,1.8,2.0$, and 2.4 for all possible atomic compositions $\left(0 \leq N_{A} \leq N\right)$. The main problem we tackle and solve here is to properly discriminate among the non-equivalent decorations of the minima. Moreover, for $R=1$ we recover the results already obtained for $2 \mathrm{D}$ and $3 \mathrm{D}$ structures with the 
Table 1: Number of local minima $\mathcal{N}_{\mathrm{LM}}$ of 3D and 2D structures, obtained with the Lennard-Jones potential. The number $1510^{*}$ was first reported in Rogan et al. [21] as well as the number of 2D minima.

\begin{tabular}{lrr}
\hline \# of atoms & $3 \mathrm{D}-\mathcal{N}_{\mathrm{LM}}$ & $2 \mathrm{D}-\mathcal{N}_{\mathrm{LM}}$ \\
\hline 5 & 1 & 1 \\
6 & 2 & 3 \\
7 & 4 & 4 \\
8 & 8 & 9 \\
9 & 21 & 16 \\
10 & 64 & 36 \\
11 & 170 & 70 \\
12 & 515 & 160 \\
13 & $1510^{*}$ & 337 \\
\hline
\end{tabular}
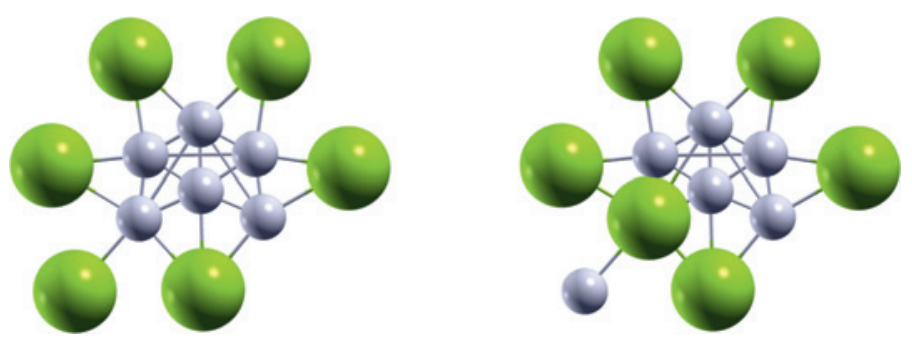

Figure 1: Two possible non-equivalent decorations of the same cluster.

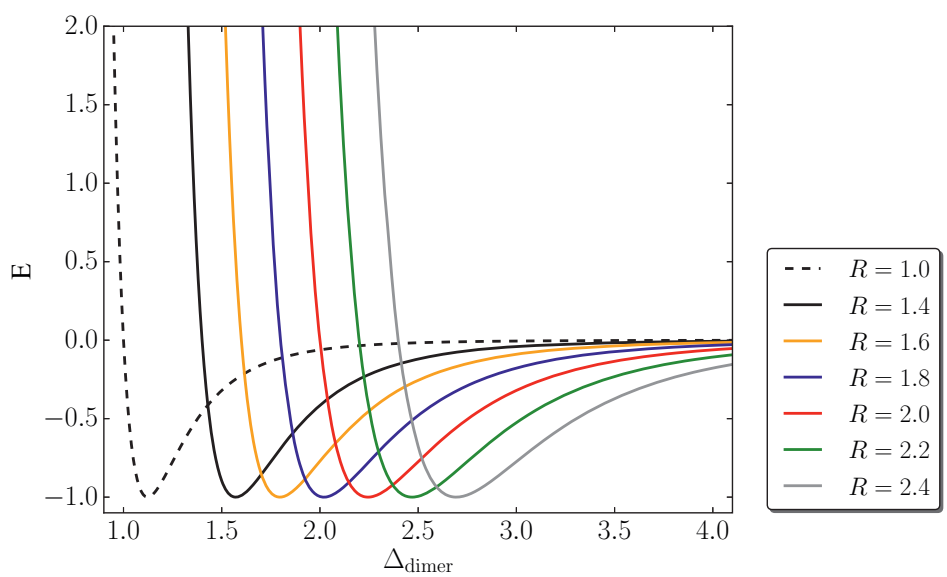

Figure 2: Plot of the total dimer energy $E$ as a function of separation of the dimer atoms $\Delta_{\text {dimer }}$. The dashed line corresponds to an $A A$ dimer; the solid lines plots correspond to $B B$ dimers for various $R$ values. Here and throughout energies are in units of $\epsilon$ and distance in units of $\sigma$. 

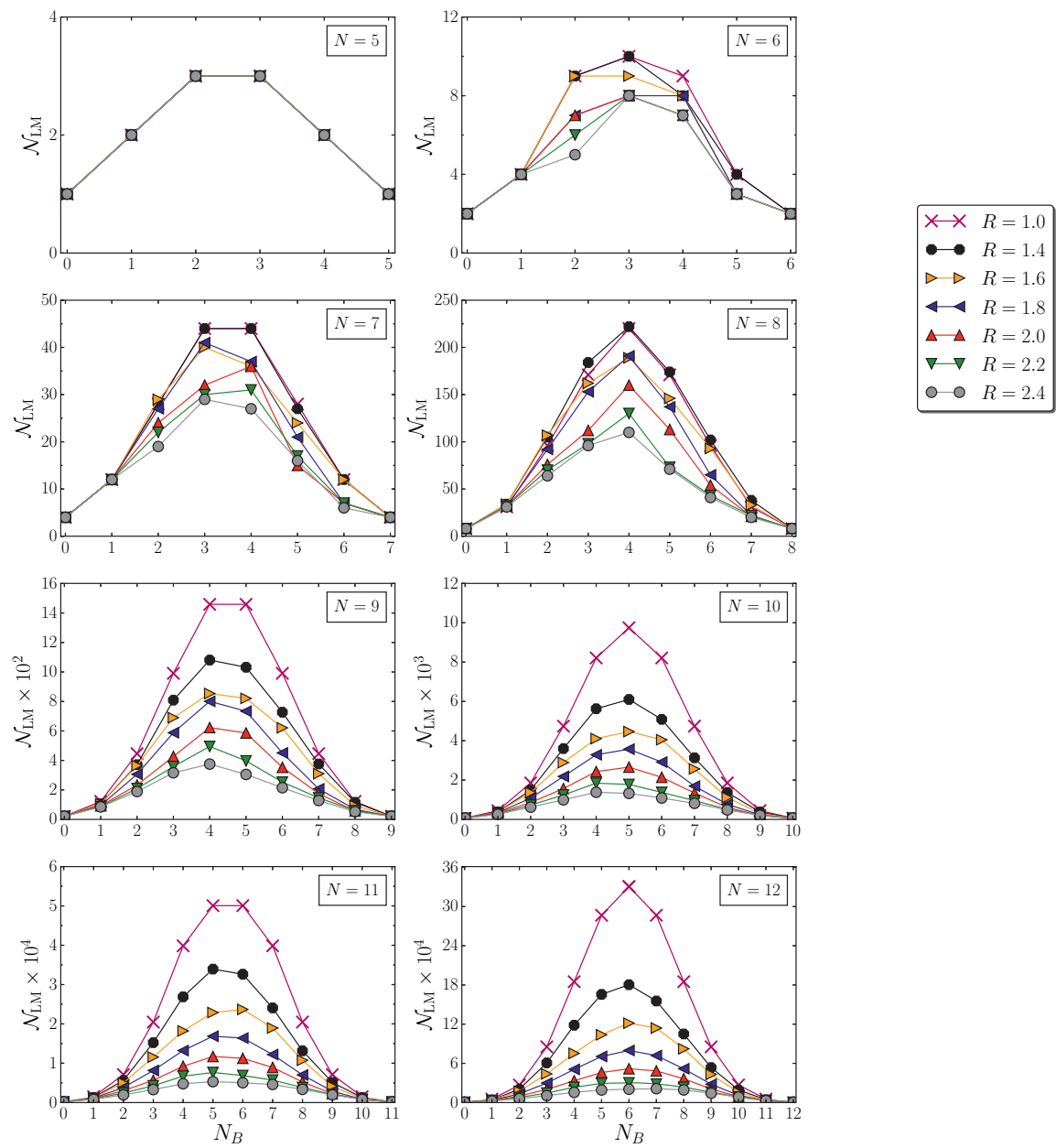

Figure 3: Number of local minima $\mathcal{N}_{\mathrm{LM}}$ of 3D BLJ structures obtained for several $R$ values, as a function of the number of $B$ atoms.

LJ potential, that are summarized on Table 1, and obtain the non-equivalent decorations. Two possible non-equivalent decorations are illustrated in Figure 1, and this underlines the importance of the proper definition of the distance concept, since the energy is not sufficient to discriminate properly.

Using the potential given by Equation 7, which essentially modifies the atomic radius of species $B$, we start by confirming the results by Takeuchi [15]. In Figure 2 we display as a reference, with dashed lines, the $R=1$ plot which corresponds to the original $\mathrm{LJ}$ potential for species $A$; the solid lines are for $B B$ dimers. The 
$R=2.0$

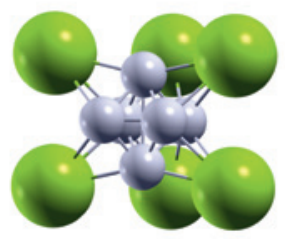

$R=2.2$

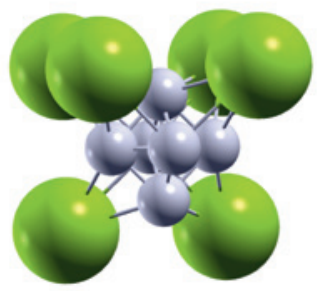

$R=2.4$

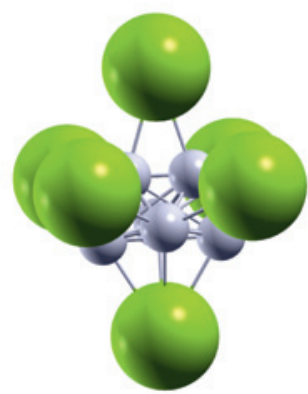

Figure 4: Minimal energy conformations for $N_{A}=N_{B}=6$, for different $R$ values. The gray spheres are $A$ type atoms. The core-shell structure is quite apparent, as well as the configuration change between $R=2.2$ and $R=2.4$.

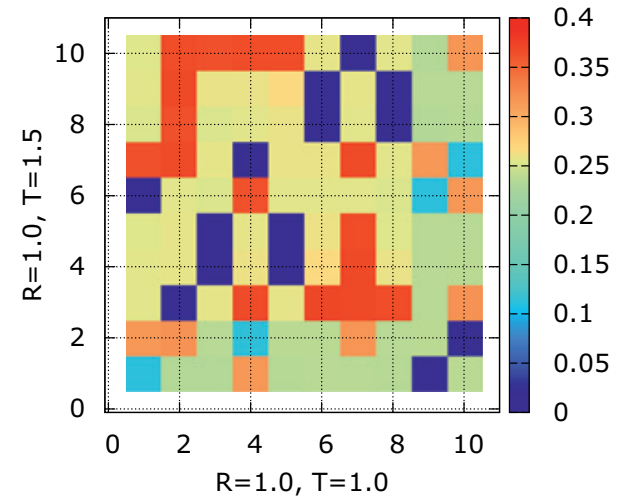

Figure 5: Illustration of the distance between the different structures, as given by the color code given on the right side, for $R=1$ and two different values of $T$.

other plots illustrate the dimer energy for other $R$ values. However, as $R$ grows, so does the range of the potential, and the position of the energy minimum [28]. The simple atomic volume change, as specified by the $R$ values, has a significant impact on the number of minima of the potential, as shown in Figure 3.

Since our focus is on the diversity Figure 3 illustrates our central result: the number of minima $\mathcal{N}_{\mathrm{LM}}$ for the all possible values of $N_{A}$ and $N_{B}$, obtained starting from random 3D conformations. The first feature that becomes apparent is that the number of minima of the BLJ structures is significantly larger than in the pure cluster case. Figure 3 also shows that as $R$ grows the number of minima decreases, as a function of $N_{B}$. Moreover, for even $N$ values the symmetry is restored as $N$ increases. 

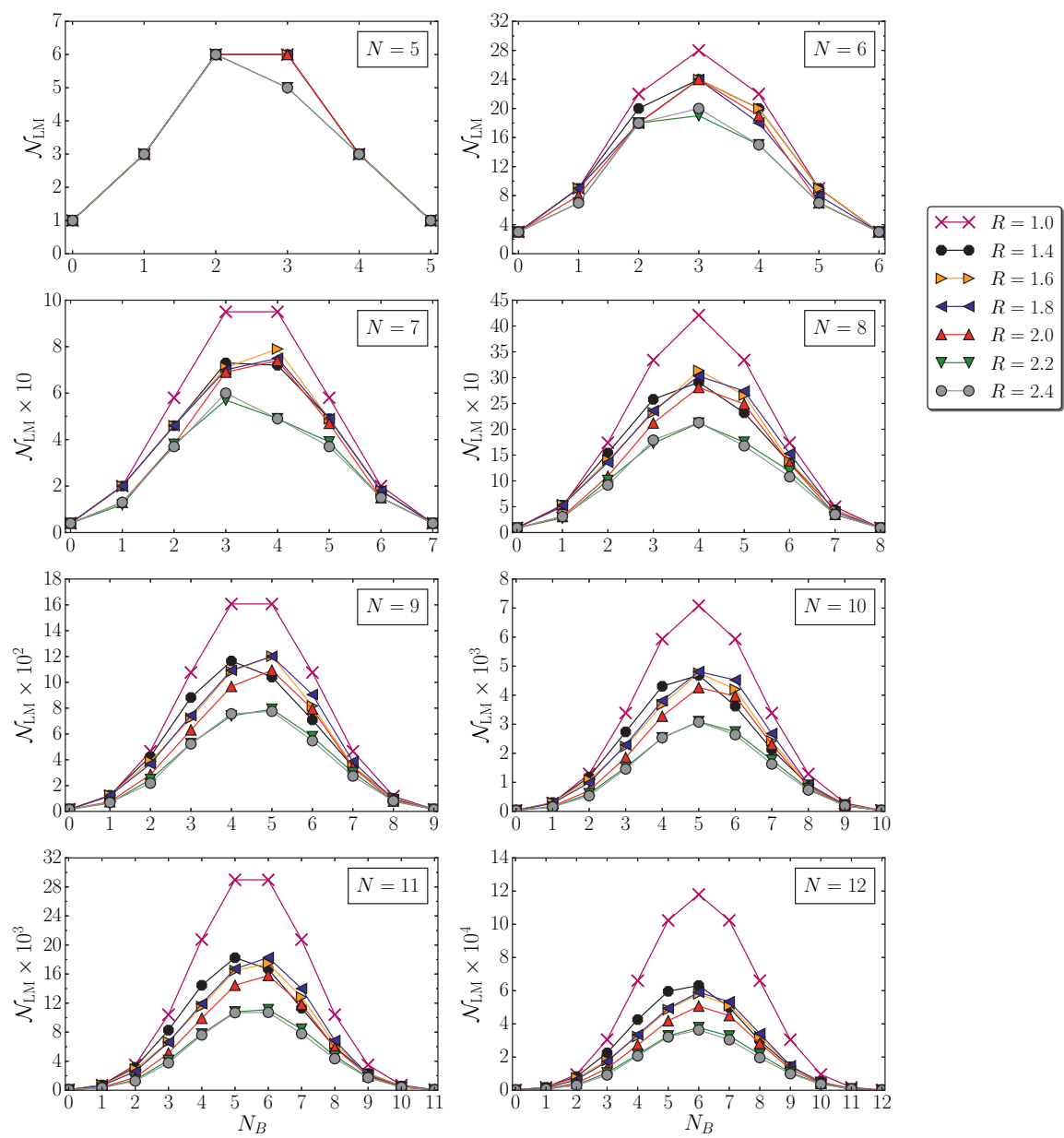

Figure 6: Number of local minima $\mathcal{N}_{\mathrm{LM}}$ of $2 \mathrm{D} \mathrm{BLJ}$ structures obtained for several $R$ values, as a function of the number of $B$ atoms. It is noticed that the non-symmetrical plot, especially notorious for $N=5$, is due to the fact that when the range of the potential varies it allows for different minima to emerge, since the minimum energy interatomic distances change.

When we compare the number of global minima with the ones in the literature they are in full agreement [15]. Moreover, we obtained a set of different minima, for each $R$, which can reach up to $10^{5}$ for $N=12$.

As mentioned above we have to use the distance between clusters concept because the cluster energy is insufficient to discriminate among minimal energy clusters. For example, for $R=1 A_{4} B_{2}$ has the same energy as $A_{2} B_{4}$, and it is the decoration that makes the difference. Here, and also from now on, we use the no- 

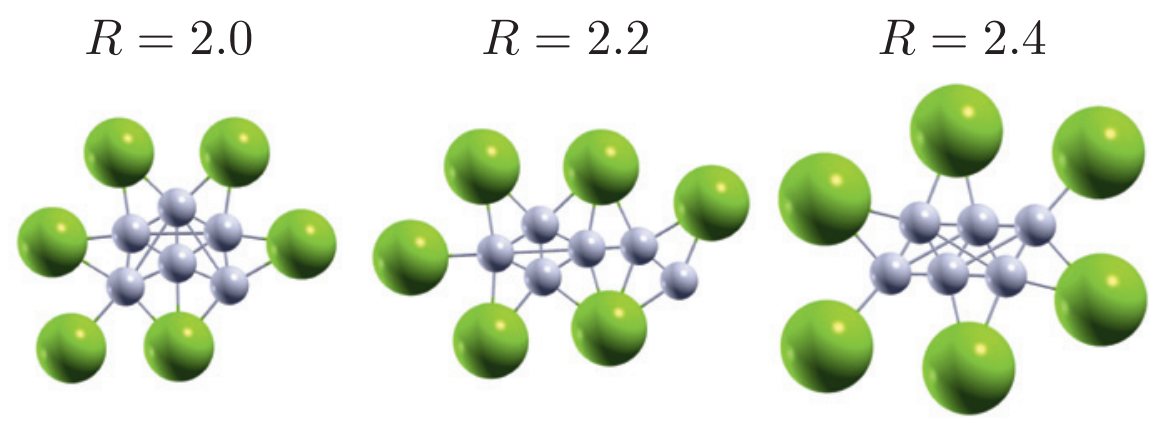

Figure 7: Top view of the minimal energy conformations of 2D structures for $N_{A}=N_{B}=6$, and several $R$ values. The gray spheres that constitute the core are $A$ type atoms. The core-shell structure is quite apparent.

tation $A_{x} B_{y}$ where $x$ is the number of $A$ atoms, and $y$ that of $B$ ones. It is worth noticing that for $R=1.4$ there are 8 minima for $A_{4} B_{2}$ and 9 for $A_{2} B_{4}$. This is related to the core-shell structure of the minimum energy conformations, which has already been reported [15]. Moreover, it is of interest that for $N \geq 10$ and for $R>2.0$ there is a variation in the maximal number of minima. In the $N=12$ case the $A$ atoms in the core change their ordering from a square base bipyrarmid to a different structure, as illustrated in Figure 4, that has an energy of 0.4 below the global $\mathrm{LJ}_{6}$ minimum.

When similar calculations are carried out for $R=1$ but $T=1.5$ the number of local minima for $N=6$ does not change. That is, the number of minima in this case is not affected by the change in the potential depth. However, the way the structures order in energy does change. This is illustrated in Figure 5 where it is observed that the same minima simply reorder, but keep their topology.

The FIRE minimization procedure is strictly local [23], and thus well suited to study planar minimal energy structures by generating at random 2D seeds, and allowing them to evolve freely. The results obtained in this way are illustrated in Figure 6. As expected, there are much less 2D than 3D minima, a result already reached by Rogan et al. [21, 22, 29, 30]. Moreover, the plots for the various $R$ values are smoother than for 3D, with less anomalies and exceptions, but keeping the core-shell arrangement, as illustrated in Figure 7. 


\section{Conclusions}

The problem of the diversity of binary nanoclusters has been investigated employing Lennard-Jones type potentials. In order to fully and precisely characterize the minimal energy conformations the energy turns out to be insufficient to discriminate among the various minima. Therefore, we introduced a generalization of the distance between cluster introduced by Grigoryan et al. [20], which is able to describe clusters of an arbitrary number of atoms and of atomic species. The diversity of the minima is obtained by a systematic examination of the set of minimal energy structures, as the BLJ $\sigma$ parameters are varied, and mainly as a function of the ratio $R$, that is given in Equation 9.

As $R$ increases the number of minima decreases, both in $2 \mathrm{D}$ and $3 \mathrm{D}$. This is due to the fact that as $R$ grows the interatomic distances also grow, and the second nearest neighbor interactions become relevant, thus adding constraints to the problem. In addition, this also implies that segregation is energetically favorable, with $A$ type atoms forming a core, and the $B$ ones a shell, consistent with the larger effective radius of the latter. This was also recently observed by Takeuchi [15]. Inspection of Figures 3 and 6 underlines the fact that the binary cluster distance concept is necessary to differentiate between equal energy conformations, like $A_{4} B_{2}$ and $A_{2} B_{4}$, for $R=1$. Moreover, we also investigated the and $R=1.0, T=1.5$ case and observed that the same minima simply reorder, but keep their topology.

Acknowledgement: This work has been founded by the Fondo Nacional de Investigaciones Científicas y Tecnológicas (FONDECYT, Chile) under Grants \#1110135 (JAV), \#1120399 and \#1130272 (MK and JR), and Financiamiento Basal para Centros Científicos y Tecnológicos de Excelencia (CEDENNA, Chile) No. FB0807.

\section{References}

1. J. Jellinek and E. B. Krissinel, Chem. Phys. Lett. 258 (1996) 283.

2. A. Varas, F. Aguilera-Granja, J. Rogan, and M. Kiwi, J. Magn. Magn. Mater. 394 (2015) 325.

3. F. Calvo and E. Yurtsever, Comput. Theor. Chem. 1021 (2013) 7.

4. J. P. K. Doye and L. Meyer, Phys. Rev. Lett. 95 (2005) 063401.

5. J. P. K. Doye and D. J. Wales, J. Phys. Chem. A 101 (1997) 5111.

6. M. Molayem, V. G. Grigoryan, and M. Springborg, J. Phys. Chem. C 115 (2011) 7179.

7. A. Cassioli, M. Locatelli, and F. Schoen, Optim. Method. Softw. 24 (2009) 819.

8. L. J. Munro, A. Tharrington, and K. J. Jordan, Comput. Phys. Commun. 145 (2002) 1.

9. J. M. Dieterich and B. Hartke, J. Comput. Chem. 32 (2010) 1377.

10. I. Kolossváry and K. J. Bowers, Phys. Rev. E 82 (2010) 056711. 
11. J. M. C. Marques and F. B. Pereira, Chem. Phys. Lett. 485 (2010) 211.

12. M. Sicher, S. Mohr, and S. Goedecker, J. Chem. Phys. 134 (2011) 044106.

13. Y. Tao, X. Ruchu, and H. Wenqi, J. Chem. Inf. Model. 51 (2011) 572, PMID: 21332209.

14. G. G. Rondina and J. L. F. Da Silva, J. Chem. Inf. Model. 53 (2013) 2282, PMID: 23957311.

15. H. Takeuchi, Comput. Theor. Chem. 1050 (2014) 68.

16. J. Lee, I. H. Lee, and J. Lee, Phys. Rev. Lett. 91 (2003) 080201.

17. J. Cheng and R. Fournier, Theor. Chem. Acc. 112 (2004) 7.

18. J. Rogan, M. Ramírez, V. Muñoz, J. A. Valdivia, G. García, R. Ramírez, and M. Kiwi, J. Phys.Condens. Mat. 21 (2009) 084209.

19. A. Sadeghi, S. A. Ghasemi, B. Schaefer, S. Mohr, M. A. Lill, and S. Goedecker, J. Chem. Phys. 139 (2013) 184118.

20. V. G. Grigoryan and M. Springborg, Chem. Phys. Lett. 375 (2003) 219.

21. J. Rogan, A. Varas, J. A. Valdivia, and M. Kiwi, J. Comput. Chem. 34 (2013) 2548.

22. J. Rogan, M. Ramírez, A. Varas, and M. Kiwi, Comput. Theor. Chem. 1021 (2013) 155.

23. E. Bitzek, P. Koskinen, F. Gähler, M. Moseler, and P. Gumbsch, Phys. Rev. Lett. 97 (2006) 170201.

24. M. Valle and A. R. Oganov, Crystal Structures Classifier for an Evolutionary Algorithm Structure Predictor, in: Visual Analytics Science and Technology, 2008, IEEE Symposium on VAST' 08 , Oct 2008, pages 11-18.

25. A. R. Oganov and M. Valle, J. Chem. Phys. 130 (2009) 104504.

26. F. Munoz, J. Rogan, G. Garcia, M. Ramírez, J. A. Valdivia, R. Ramírez, and M. Kiwi. Eur. Phys. J. D 61 (2011) 87.

27. F. Munoz, J. Rogan, J. A. Valdivia, A. Varas, and M. Kiwi, Physica B 427 (2013) 76.

28. J. P. K. Doye, D. J. Wales, and R. S. Berry, J. Chem. Phys. 103 (1995) 170201.

29. J. H. Conway and N. J. A. Sloane, Sphere Packings, Lattices and Groups, Springer, New York (1988).

30. J. H. Conway and N. J. A. Sloane, Sphere Packings, Lattices and Groups, Springer, Berlin (1998). 